\title{
APPLICATION OF A SUPERPARAMETRIC FINITE SHELL ELEMENT TO THE CALCULATION OF TURBINE BLADE VIBRATIONS
}

\author{
S. I. Bogomolov, S. S. Lutsenko, S. A. Nazarenko
}

Determination of the natural frequencies and forms of vibrations of turbine blades with the present level of knowledge is one of the chief problems that have to be solved at the design stage of turbines in order to ensure vibration reliability. The more stringent requirements that the mechanical properties of critical units, especially blades, have to fulfill when subjected to vibrations make it necessary to investigate the high-frequency part of the spectrum to which correspond the "lamellar" forms of vibrations, Moreover, the lamellar forms are a characteristic feature (even at low frequencies) of blades whose axial and transverse dimensions are commensurable.

The most widely used calculation schemes of blades - treating them as rods - do not make it possible to determine the natural vibration frequencies corresponding to such forms with the required accuracy. Greater possibilities of devising more correct mathematical models of blades and universal methods of investigating their vibrations are opened up in connection with the application of the finite element method.

In the present work we use a superparametric shell element obtained from the full three-dimensional isoparametric form by introducing the hypotheses that characterize the mathematical models of Timoshenko shells. The outstanding suitability of the element is due to the fact that the hypotheses concerning the state of stress and strain of shells are taken into account already during the process of constructing the element. Therefore, to obtain the matrix characteristics of the element, it is expedient to use the basic relationships of the theory of elasticity. In essence, the element is intermediate between two dimensional curvilinear shell elements and three-dimensional curvilinear elements, and this makes it possible to overcome the basic difficulties that are typical of the finite elements of other types in checking blades for dynamic strength.

The superparametric element used, having 40 degrees of freedom, was obtained from a rectangular parabolic base element and belongs to the serendipitous family. The algorithm for obtaining the chief characteristics of the element --matrices of rigidity and matrices of masses -- was explained in [1-3]. It should only be pointed out that, regardless of the simple integration limits, the expressions for the integrands are complex and, therefore, it is necessary to integrate numerically in order to obtain the matrices of rigidity and of masses. Most effective is the use of Gauss' formula of integration. And numerous calculations $[1,2]$ showed that the use of the formula by one order of magnitude lower (lowered order of integration -- LOI) than necessary, in accordance with the order of the approximating polynomial (basic rule of integration - BRI), makes it possible to improve substantially the results of the calculation. 
It is known that the dynamic calculation of finite element models reduces to the investigation of an algebraic system of equations whose matrix has a strip like structure. For its construction the matrix of indices [4] is used; natural frequencies and forms of vibrations are determined by the square root method [5]. The algorithm of the calculation was realized in the complex of FORTRAN programs for BESM-6 computers.

Thus the suggested approach yields good agreement between calculation and experiment. Here an important part is played by the use of accurate and not approximate methods of investigating the resolving algebraic system of equations, and also by the adequate selection of nodal points with whose aid the geometry of the finite elements is specified.

\section{LITERATURE CITED}

1. S. Ahmad, B. M. Irons, and O. C. Zienkiewicz, "Analysis of thick and thin shell structures by curved finite elements," Int. J. Num. Meth. Eng., 2, No. 4, 419-451 (1970).

2. O. C. Zienkiewicz, R. L. Taylor, and J. M. Too, "Reduced integration technique in general analysis of plates and shells," Int. J. Num. Meth. Eng., 3, No. 2, 275-290 (1971).

3. S. Ahmad, R. G. Anderson, and O. C. Zienkiewicz, "Vibration of thick curved shells, with particular reference to turbine blades," J. Strain Anal., No. 3, 200-206 (1970).

4. V. A. Postnov and I. Ya. Kharkhurim, The Finite Element Method in the Calculation of Ship Structures [in Russian], Sudostroenie, Leningrad (1974).

5. V. V. Voevodin, Numerical Methods of Algebra: Theory and Algorithms [in Russian], Fizmatgiz, Moscow (1966).

6. M. D. Olson and G. M. Lindberg, "Vibration analysis of cantilevered curved plates, using a new cylindrical shell, finite element," in: Proc. 2nd Conf. Matr. Meth. Struct.

Mech., AF Base Wright-Patterson, Ohio (1968), pp. 247-269.

7. G. R. Cowper, G. M. Lindberg, and M. D. Olson, "A shallow shell finite element of triangular shape," Int. J. Solids Struct., 6, No. 12, 1133-1156 (1970).

8. K. P. Walker, "Vibrations of cambered helicoidally fan blades," J. Sound Vib., 59, No. 1, 35-57 (1978).

9. M. A. Bossak and O. C. Zienkiewicz, "Free vibration of initially stressed solids with particular reference to centrifugal force in rotating machinery," J. Strain Anal., 8, No. 4, 245-252 (1973).

10. L. D. Hofmeister and D. A. Evensen, "Vibration problems using isoparametric shell elements," Int. J. Num. Meth. Eng., No. 1, 142-145 (1972). 